\title{
Synthesis of Quinoline $\mathrm{N}$-Oxides from the Baylis-Hillman Adducts of 2-Nitrobenzaldehydes: Conjugate Addition of Nitroso Intermediate
}

\author{
Ka Young Lee, Seung Chan Kim, and Jae Nyoung Kim* \\ Department of Chemistry and Institute of Basic Science, Chonnam National University, Gwangju 500-757, Korea \\ *E-mail:kimjn@chonnam.ac.kr \\ Received April 18, 2005
}

Key Words : Quinoline $N$-oxides, Baylis-Hillman adducts, 2-Nitrobenzaldehydes, Zinc, Ammonium chloride

The Baylis-Hillman reaction is a useful carbon-carbon bond-forming method from activated vinyls and carbonyl compounds. ${ }^{1}$ Chemical transformation of the BaylisHillman adducts or their derivatives into useful heterocyclic compounds has been studied recently by us and other groups. ${ }^{1-3}$ Especially, conversion of the Baylis-Hillman adducts derived from 2-nitrobenzaldehydes into quinoline skeleton is a useful entry for the quinoline chemistry. ${ }^{20,3,4}$

Kaye and co-workers have reported the synthesis of quinoline skeleton from the Baylis-Hillman adducts. ${ }^{3 \mathrm{~b}}$ We have also reported the synthesis of 4-hydroxyquinoline $\mathrm{N}$ oxides from the Baylis-Hillman adducts of 2-nitrobenzaldehydes. $^{20,3 a}$ As a continuing study, we examined the synthesis of quinoline $N$-oxides 2 by reduction of the nitro functionality of the Baylis-Hillman adducts $\mathbf{1}$ into nitroso group followed by dehydrative cyclization strategy. Among the various examined reduction conditions, ${ }^{4}$ the use of zinc and ammonium chloride system in aqueous THF was found to be effective.

The nitro group is readily converted to a series of functions of various degrees of reduction: very exceptionally to a nitroso group, more often to a hydroxylamino group and most frequently to the amino group. ${ }^{5}$ Nitroso compounds are usually not obtained directly but rather by reoxidation of hydroxylamino compounds, ${ }^{5}$ which can be prepared from nitro compounds by zinc and aqueous ammonium chloride. ${ }^{6}$ Moreover there have been few instances in which nitroso compounds have been isolated as intermediates in reductions of nitro compounds. ${ }^{5,7}$ In some instances the nitroso nitrogen behaves as a nucleophile with formaldehyde, glyoxylic acid and pyruvic acid. ${ }^{8}$ Only one example of the conjugate addition of arylnitroso compound to the conjugated azoalkene system was known to the best of our knowledge. ${ }^{9}$

The reaction of the Baylis-Hillman adduct 1a and zinc (2.0 equiv) and ammonium chloride ( 2.0 equiv) in aqueous THF at $60-70{ }^{\circ} \mathrm{C}$ gave the quinoline $N$-oxide $2 \mathbf{a}$ in $49 \%$ isolated yield (Scheme 1) together with many intractable side products. In the reaction, quenching of the unstable nitroso intermediate (I) occurred before further reduction to a hydroxylamine stage due to the facile intramolecular Michael addition process. This might be the first example of conjugate addition of the nitroso intermediate to the Michael acceptor in an intramolecular fashion as mentioned

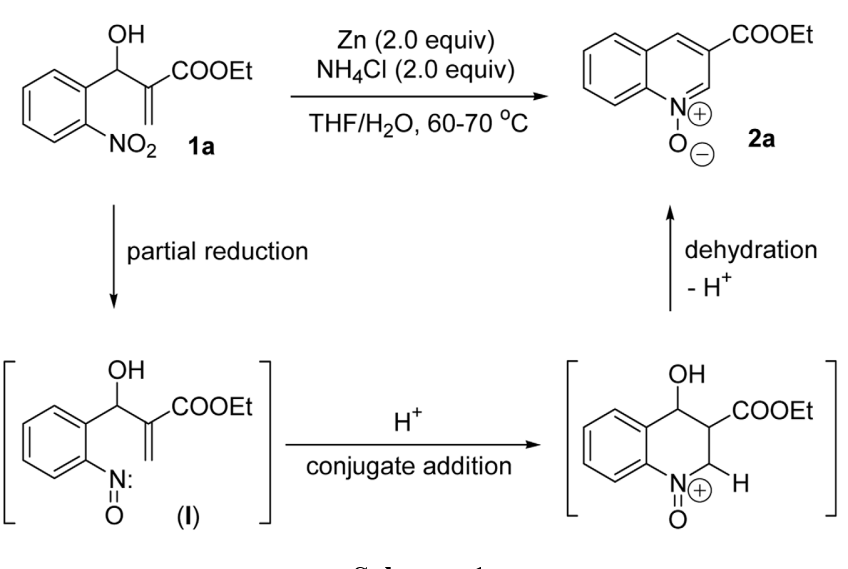

Scheme 1

Table 1. Synthesis of quinoline N-oxidex 2a-e

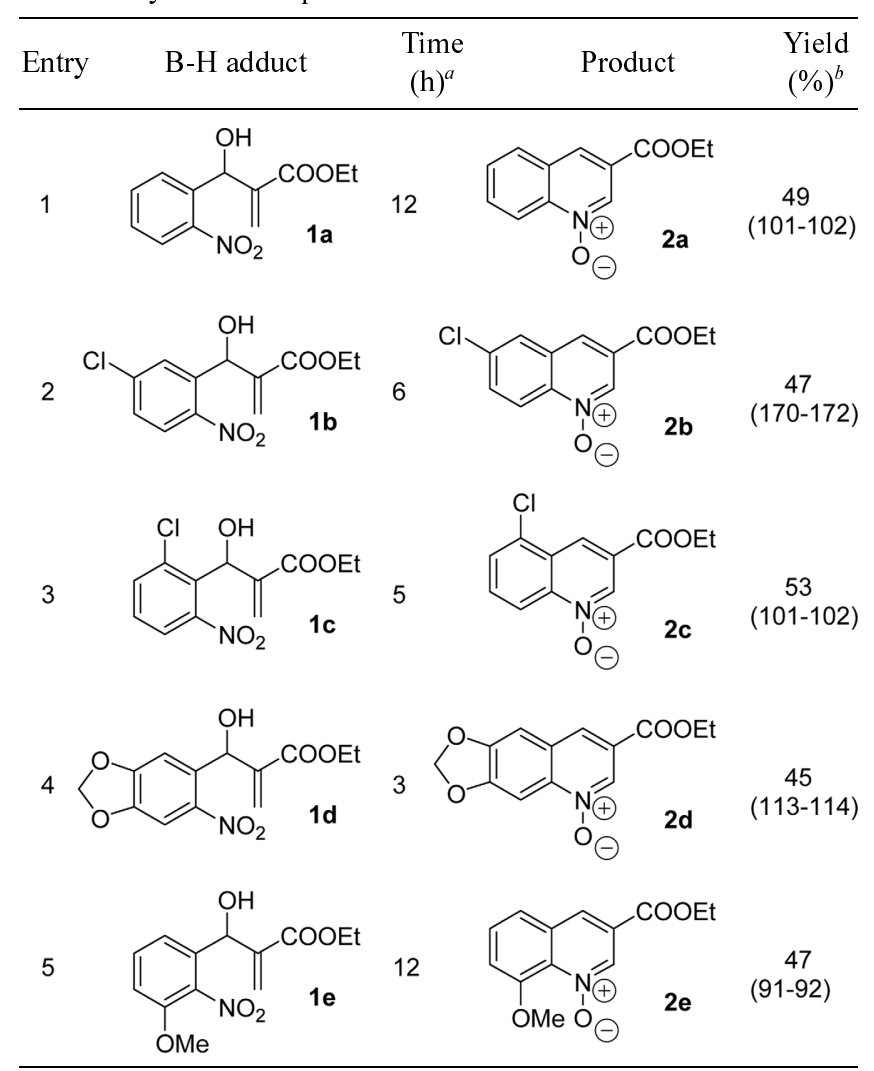

${ }^{a}$ Reaction conditions: $\mathrm{Zn}$ (2.0 equiv), $\mathrm{NH}_{4} \mathrm{Cl}$ (2.0 equiv), THF- $\mathrm{H}_{2} \mathrm{O}, 60-$ $70{ }^{\circ} \mathrm{C} .{ }^{b} \mathrm{Mp}$ was written in parenthesis. 


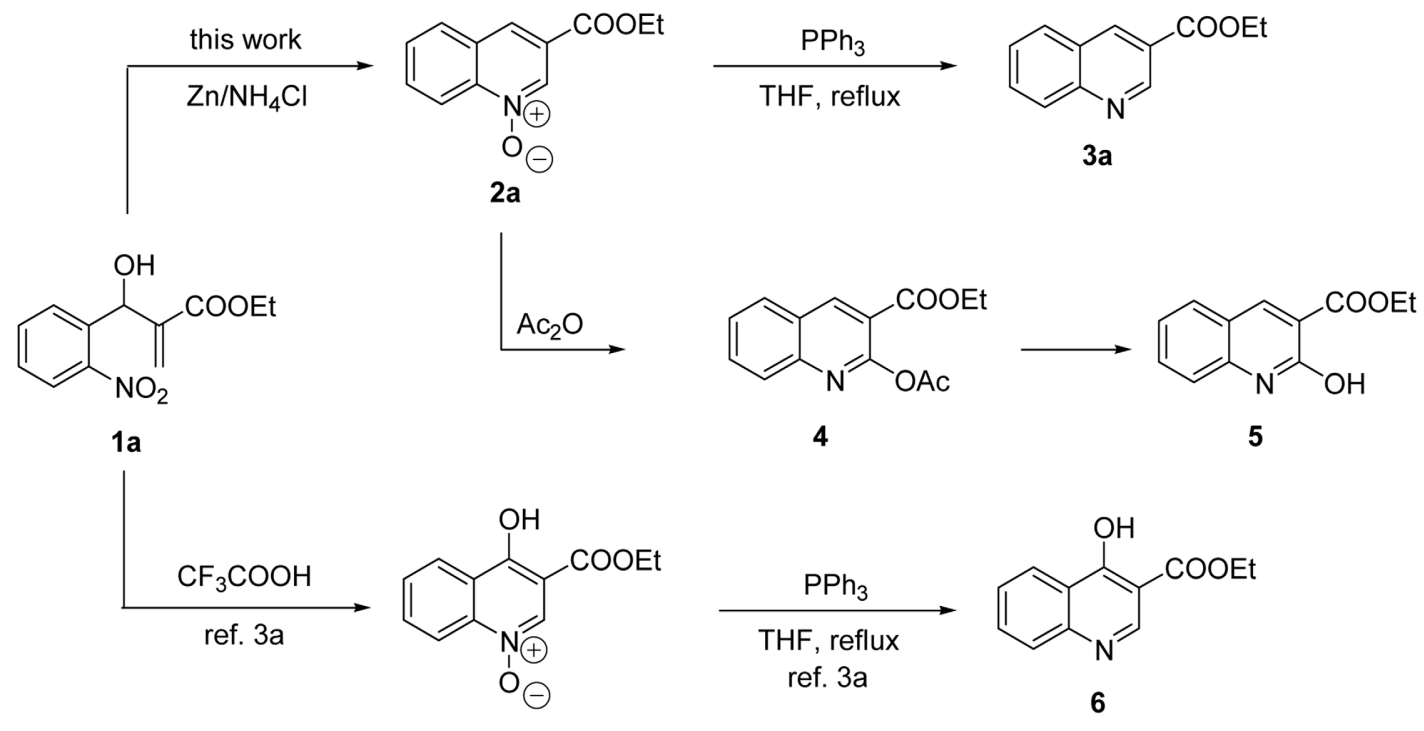

Scheme 2

before. $^{20,3 a}$ The representative results are summarized in Table 1.

The reaction mechanism is thought to be as follows as shown in Scheme 1: (1) Reduction of the nitro functionality of 1 to the nitroso group to give (I), (2) acid catalyzed intramolecular conjugate addition, and finally (3) dehydration gave the products 2 . The structure of $\mathbf{2 a}$ can be easily confirmed as shown in Scheme 2. Deoxygenation of 2a with triphenylphosphine afforded the corresponding quinoline $\mathbf{3 a}$ (THF, $\mathrm{PPh}_{3}, 48 \mathrm{~h}, 70 \%$ ), which was identical with the authentic sample prepared according to our previous method from the Baylis-Hillman adduct of 2-fluorobenzaldehyde $N$ tosylimine. ${ }^{2 p}$ The reaction of $\mathbf{2 a}$ with acetic anhydride gave 2-acetoxy derivative 4 in 58\% yield as reported in a similar system. ${ }^{10}$ It is interesting to note that the acetoxy compound 4 was unstable and converted spontaneously to the 2hydroxyquinoline derivative $\mathbf{5}^{11}$ slowly. As exemplified in Scheme 2, regioselective synthesis of 2-hydroxyquinoline $\mathbf{5}$ (via successive treatment of $\mathbf{1 a}$ with $\mathrm{Zn} / \mathrm{NH}_{4} \mathrm{Cl}$ and $\mathrm{Ac}_{2} \mathrm{O}$ ) and 4-hydroxyquinoline 6 (via successive treatment of $\mathbf{1 a}$ with $\mathrm{CF}_{3} \mathrm{COOH}$ and $\left.\mathrm{PPh}_{3}\right)^{3 \mathrm{a}}$ could be carried out. ${ }^{12}$ Partial deoxygenation of quinoline $N$-oxide to quinoline was observed after long reaction time under the reduction conditions in electron-rich system such as $\mathbf{2 d}{ }^{13}$

In summary, we disclosed on the facile one-pot preparation method of quinoline $N$-oxides from the BaylisHillman adducts of 2-nitrobenzaldehydes via the conjugate addition of the nitroso functionality in an intramolecular fashion as the key step for the first time.

\section{Experimental Section}

Typical procedure for the synthesis of $2 a$. To a stirred suspension of 1a $(251 \mathrm{mg}, 1.0 \mathrm{mmol})$, zinc $(130 \mathrm{mg}, 2.0$ $\mathrm{mmol})$ and ammonium chloride $(110 \mathrm{mg}, 2.0 \mathrm{mmol})$ in aqueous THF $\left(4 \mathrm{~mL}, \mathrm{THF} / \mathrm{H}_{2} \mathrm{O}=3: 1\right)$ was heated to $60-70$ ${ }^{\circ} \mathrm{C}$ for $12 \mathrm{~h}$. After filtration through a Celite pad, appropriate workup process and column chromatographic purification (hexanes/ethyl acetate $=1: 1$ ) 2a was obtained as a white solid, $107 \mathrm{mg}$ (49\%). Compounds 2b-e was prepared similarly and their spectroscopic data are as follows.

Compound 2a: white solid, mp 101-102 ${ }^{\circ} \mathrm{C}$; IR (KBr) 1724, 1374, 1364, 1276, 1247, 1207, $770 \mathrm{~cm}^{-1}$; ${ }^{1} \mathrm{H}$ NMR $\left(300 \mathrm{MHz}, \mathrm{CDCl}_{3}\right) \delta 1.45(\mathrm{t}, J=7.2 \mathrm{~Hz}, 3 \mathrm{H}), 4.47(\mathrm{q}, J=7.2$ $\mathrm{Hz}, 2 \mathrm{H}), 7.43$ (t, $J=8.1 \mathrm{~Hz}, 1 \mathrm{H}), 7.89$ (t, $J=8.4 \mathrm{~Hz}, 1 \mathrm{H})$, $8.01(\mathrm{~d}, J=8.1 \mathrm{~Hz}, 1 \mathrm{H}), 8.43(\mathrm{~s}, 1 \mathrm{H}), 8.79(\mathrm{~d}, J=8.4 \mathrm{~Hz}$, 1H), $9.09(\mathrm{~s}, 1 \mathrm{H}) ;{ }^{13} \mathrm{C} \mathrm{NMR}\left(75 \mathrm{MHz}, \mathrm{CDCl}_{3}\right) \delta 14.16$, $62.13,119.91,124.61,127.82,129.11,129.35,129.49$, 132.48, 135.13, 142.91, 163.31; Mass (CI) $\mathrm{m} / z$ (rel. intensity) 116 (14), 128 (16), 156 (30), 173 (17), 189 (19), 202 (68), $217\left(\mathrm{M}^{+}, 90\right), 218\left(\mathrm{MH}^{+}, 100\right)$.

Compound $2 \mathbf{b}$ : white solid, mp 170-172 ${ }^{\circ} \mathrm{C}$; ${ }^{1} \mathrm{H}$ NMR $(300$ $\left.\mathrm{MHz} \mathrm{CDCl}_{3}\right) \delta 1.45(\mathrm{t}, J=7.2 \mathrm{~Hz}, 3 \mathrm{H}), 4.48(\mathrm{q}, J=7.2 \mathrm{~Hz}$, 2H), $7.79(\mathrm{dd}, J=9.3$ and $2.1 \mathrm{~Hz}, 1 \mathrm{H}), 7.98(\mathrm{~d}, J=2.1 \mathrm{~Hz}$, 1H), $8.31(\mathrm{~s}, 1 \mathrm{H}), 8.72(\mathrm{~d}, J=9.3 \mathrm{~Hz}, 1 \mathrm{H}), 9.02$ (d, $J=1.2$ $\mathrm{Hz}, 1 \mathrm{H}) ;{ }^{13} \mathrm{C}$ NMR $\left(75 \mathrm{MHz}, \mathrm{CDCl}_{3}\right) \delta 14.20,62.37$, $121.92,126.00,126.26,128.13,130.02,133.07,135.24$, 135.91, 141.60, 163.01.

Compound 2c: white solid, mp 101-102 ${ }^{\circ} \mathrm{C}$; ${ }^{1} \mathrm{H}$ NMR (300 $\left.\mathrm{MHz}, \mathrm{CDCl}_{3}\right) \delta 1.47(\mathrm{t}, J=7.2 \mathrm{~Hz}, 3 \mathrm{H}), 4.50(\mathrm{q}, J=7.2 \mathrm{~Hz}$, 2H), 7.77-7.80 (m, 2H), $8.71(\mathrm{dd}, J=7.5$ and $2.1 \mathrm{~Hz}, 1 \mathrm{H})$, $8.76(\mathrm{~s}, 1 \mathrm{H}), 9.08(\mathrm{~d}, J=0.9 \mathrm{~Hz}, 1 \mathrm{H}) ;{ }^{13} \mathrm{C} \mathrm{NMR}(75 \mathrm{MHz}$, $\left.\mathrm{CDCl}_{3}\right) \delta 14.21,62.40,119.12,124.13,125.33,127.79$, $129.73,131.86,133.85,135.57,144.14,163.06$.

Compound 2d: white solid, mp 113-114 ${ }^{\circ} \mathrm{C} ;{ }^{1} \mathrm{H}$ NMR (300 $\left.\mathrm{MHz}, \mathrm{CDCl}_{3}\right) \delta 1.43(\mathrm{t}, J=7.2 \mathrm{~Hz}, 3 \mathrm{H}), 4.44(\mathrm{q}, J=7.2 \mathrm{~Hz}$, 2H), $6.23(\mathrm{~s}, 2 \mathrm{H}), 7.20(\mathrm{~s}, 1 \mathrm{H}), 8.12(\mathrm{~s}, 1 \mathrm{H}), 8.23(\mathrm{~s}, 1 \mathrm{H})$, $8.95(\mathrm{~d}, J=1.5 \mathrm{~Hz}, 1 \mathrm{H}) ;{ }^{13} \mathrm{C}$ NMR $\left(75 \mathrm{MHz}, \mathrm{CDCl}_{3}\right) \delta$ 14.22, 61.98, 97.91, 102.90, 104.35, 123.23, 126.58, 126.69, $134.57,141.28,150.15,153.60,163.55$.

Compound 2e: white solid, mp 91-92 ${ }^{\circ} \mathrm{C}$; ${ }^{1} \mathrm{H}$ NMR (300 $\left.\mathrm{MHz}, \mathrm{CDCl}_{3}\right) \delta 1.45(\mathrm{t}, J=7.2 \mathrm{~Hz}, 3 \mathrm{H}), 4.12(\mathrm{~s}, 3 \mathrm{H}), 4,49$ (q, $J=7.2 \mathrm{~Hz}, 2 \mathrm{H}), 7.18(\mathrm{dd}, J=7.2$ and $1.8 \mathrm{~Hz}, 1 \mathrm{H}), 7.51-$ $7.56(\mathrm{~m}, 2 \mathrm{H}), 8.82$ (d, $J=2.1 \mathrm{~Hz}, 1 \mathrm{H}), 9.45$ (d, $J=2.1 \mathrm{~Hz}$, 
1H).

Conversion of 2a into 5. A solution of 2a (217 $\mathrm{mg}, 1$ mmol) in acetic anhydride $(1 \mathrm{~mL})$ was heated to reflux for 4 h. After usual workup and column chromatographic purification (hexanes/ether, $1: 1$ ) process we obtained 4 as a white solid, $150 \mathrm{mg}$ (58\%). However, the compound 4 was unstable and converted into spontaneously to $\mathbf{5}$. After 7 days at room temperature we found quantitative formation of $\mathbf{5}$. Analytically pure sample $\mathbf{5}$ was obtained by flash column chromatography (hexanes/ether, $1: 1)$ in $82 \%$ yield (178 $\mathrm{mg})$. The spectroscopic data of $\mathbf{4}$ and $\mathbf{5}$ are as follows.

Compound 4: ${ }^{1} \mathrm{H}$ NMR (300 MHz, $\left.\mathrm{CDCl}_{3}\right) \delta 1.43(\mathrm{t}, J=$ $7.2 \mathrm{~Hz}, 3 \mathrm{H}), 2.45(\mathrm{~s}, 3 \mathrm{H}), 4.42(\mathrm{q}, J=7.2 \mathrm{~Hz}, 2 \mathrm{H}), 7.62$ (t, $J$ $=8.1 \mathrm{~Hz}, 1 \mathrm{H}), 7.84(\mathrm{t}, J=8.1 \mathrm{~Hz}, 1 \mathrm{H}), 7.95(\mathrm{~d}, J=8.1 \mathrm{~Hz}$, $1 \mathrm{H}), 8.04(\mathrm{~d}, J=8.1 \mathrm{~Hz}, 1 \mathrm{H}), 8.96(\mathrm{~s}, 1 \mathrm{H})$.

Compound 5: white solid, mp 162-163 ${ }^{\circ} \mathrm{C}$; ${ }^{1} \mathrm{H}$ NMR (300 $\left.\mathrm{MHz}, \mathrm{CDCl}_{3}\right) \delta 1.45(\mathrm{t}, J=7.2 \mathrm{~Hz}, 3 \mathrm{H}), 4.46(\mathrm{q}, J=7.2 \mathrm{~Hz}$, $2 \mathrm{H}), 7.26(\mathrm{t}, J=8.4 \mathrm{~Hz}, 1 \mathrm{H}), 7.50(\mathrm{~d}, J=8.4 \mathrm{~Hz}, 1 \mathrm{H}), 7.62$ $(\mathrm{t}, J=8.4 \mathrm{~Hz}, 1 \mathrm{H}), 7.66(\mathrm{~d}, J=8.4 \mathrm{~Hz}, 1 \mathrm{H}), 8.57(\mathrm{~s}, 1 \mathrm{H}) ;{ }^{13} \mathrm{C}$ NMR $\left(75 \mathrm{MHz}, \mathrm{CDCl}_{3}\right) \delta 14.31,61.45,116.20,118.61$, $122.18,123.19,129.19$, 133.08, 139.98, 145.67, 161.14, 164.39 .

The spectroscopic data of $3 \mathrm{a}^{2 \mathrm{p}}$ and $\mathbf{6}^{3 \mathrm{a}}$ can be found in our previous papers.

Acknowledgments. This work was supported by the grant (R-05-2003-000-10042-0) from the Basic Research Program of the Korea Science and Engineering Foundation (Now controlled under the authority of Korea Research Foundation). Spectroscopic data was obtained from the Korea Basic Science Institute, Gwangju branch.

\section{References and Notes}

1. For the review articles of Baylis-Hillman reaction, see (a) Basavaiah, D.; Rao, P. D.; Hyma, R. S. Tetrahedron 1996, 52, 8001. (b) Ciganek, E. Organic Reactions; John Wiley \& Sons: New York, 1997; Vol. 51, pp 201-350. (c) Drewes, S. E.; Roos, G. H. P. Tetrahedron 1988, 44, 4653. (d) Langer, P. Angew. Chem., Int. Ed. Engl. 2000, 39, 3049. (e) Kim, J. N.; Lee, K. Y. Curr. Org. Chem. 2002, 6, 627. (f) Basavaiah, D.; Rao, A. J.; Satyanarayana, T. Chem. Rev. 2003, 103, 811.

2. For our recent papers on the synthesis of heterocyclic compounds from Baylis-Hillman adducts, see (a) Lee, C. G.; Lee, K. Y.; Kim, S. C.; Kim, J. N. Bull Korean Chem. Soc. 2005, 26, 485. (b) Lee, C. G.; Gowrisankar, S.; Kim, J. N. Bull. Korean Chem. Soc. 2005, 26, 481. (c) Lee, M. J.; Lee, K. Y.; Kim, J. N. Bull. Korean Chem.
Soc. 2005, 26, 477. (d) Gowrisankar, S.; Na, J. E.; Lee, M. J.; Kim, J. N. Bull. Korean Chem. Soc. 2005, 26, 319. (e) Lee, C. G.; Lee, K. Y.; Lee, S.; Kim, J. N. Tetrahedron 2005, 61, 1493. (f) Lee, K. Y.; Kim, T. H.; Kim, J. N. Bull. Korean Chem. Soc. 2004, 25, 1966. (g) Gowrisankar, S.; Lee, C. G.; Kim, J. N. Tetrahedron Lett. 2004, 45, 6949. (h) Gowrisankar, S.; Lee, K. Y.; Lee, C. G.; Kim, J. N. Tetrahedron Lett. 2004, 45, 6141. (i) Lee, K. Y.; Gowrisankar, S.; Kim, J. N. Tetrahedron Lett. 2004, 45, 5485. (j) Kim, J. M.; Lee, K. Y.; Lee, S.-k.; Kim, J. N. Tetrahedron Lett. 2004, 45, 2805. (k) Lee, K. Y.; Kim, J. M.; Kim, J. N. Tetrahedron Lett. 2003, 44, 6737. (1) Kim, J. N.; Kim, J. M.; Lee, K. Y. Synlett 2003, 821. (m) Im, Y. J.; Lee, C. G.; Kim, H. R.; Kim, J. N. Tetrahedron Lett. 2003, 44, 2987. (n) Lee, K. Y.; Kim, J. M.; Kim, J. N. Synlett 2003, 357. (o) Lee, K. Y.; Kim, J. M.; Kim, J. N. Tetrahedron 2003, 59, 385. (p) Kim, J. N.; Lee, H. J.; Lee, K. Y.; Kim, H. S. Tetrahedron Lett. 2001, 42, 3737.

3. (a) Kim, J. N.; Lee, K. Y.; Kim, H. S.; Kim, T. Y. Org. Lett. 2000, 2, 343. (b) Familoni, O. B.; Kaye, P. T.; Klaas, P. J. J. Chem. Soc., Chem. Commun. 1998, 2563.

4. The use of other reducing conditions provided somewhat different products. For example, the use of zinc or iron in acidic media gave $2(1 H)$-quinolinone derivatives. The use of $\mathrm{SnCl}_{2}$ in alcoholic solvent gave the similar compounds, 3-alkoxymethyl 2(1H)quinolinones. Please see the following references: (a) Lee, K. Y.; Kim, J. N. Bull. Korean Chem. Soc. 2002, 23, 939. (b) Lee, K. Y.; Kim, J. M.; Kim, J. N. Bull. Korean Chem. Soc. 2002, 23, 1493.

5. Kabalka, G. W.; Varma, R. S. In Comprehensive Organic Synthesis; Trost, B. M.; Fleming, I., Eds.; Oxford: Pergamon, 1991; Vol 8, pp 363-379.

6. (a) Kamm, O. Org. Syn. Coll; 1932; Vol. 1, p 445. (b) Patrick, T. B.; Schield, J. A.; Kirchner, D. G. J. Org. Chem. 1974, 39, 1758.

7. (a) Ross, S. D.; Kahan, G. J.; Leach, W. A. J. Am. Chem. Soc. 1952, 74, 4122. (b) Ross, S. D.; Kuntz, I. J. Am. Chem. Soc. 1952 $74,1297$.

8. (a) Kronja, O.; Matijevic-Sosa, J.; Ursic, S. J. Chem. Soc., Chem. Commun. 1987, 463. (b) Pilepic, V.; Ursic, S. Tetrahedron Lett. 1994, 35, 7425. (c) Corbett, M. D.; Corbett, B. R. J. Org. Chem. 1980, 45, 2834. (d) Ursic, S.; Pilepic, V.; Vrcek, V.; Gabricevic, M.; Zorc, B. J. Chem. Soc., Perkin Trans. 2 1993, 509.

9. Attanasi, O. A.; Filippone, P.; Fiorucci, C. Tetrahedron 1997, 53, 1467.

10. Boekelheide, V.; Linn, W. J. J. Am. Chem. Soc. 1954, 76, 1286.

11. (a) Coutts, R. T.; Wibberley, D. G. J. Chem. Soc. 1962, 2518. (b) Suzuki, M.; Ohuchi, Y.; Asanuma, H.; Kaneko, T.; Yokomori, S.; Ito, C.; Isobe, Y.; Muramatsu, M. Chem. Pharm. Bull. 2000, 48, 2003.

12. Studies on the regioselective synthesis of 2-hydroxy- and 4hydroxyquinoline derivatives are under progress and will be published in due course.

13. We could isolate the corresponding deoxygenated compound as the major (43\%) when we performed the reaction of $1 \mathrm{~d}$ for $20 \mathrm{~h}$. For deoxygenation with $\mathrm{Zn} / \mathrm{NH}_{4} \mathrm{Cl}$ in a similar system, see. Aoyagi, Y.; Abe, T.; Ohta, A. Synthesis 1997, 891. 